\title{
The Standardized Research and Teaching Guidance on the Introduction of the Japanese Language in Xi'an Tourist Attractions
}

\author{
Tie Man \\ Institute of Foreign Language the Xi'an university, 710065
}

\begin{abstract}
Keywords: Xi'an, tourist attractions, Japanese translation, Japanese introduction, Japanese Language Teaching
\end{abstract}

\begin{abstract}
Japan remains an important source of visitors to $\mathrm{Xi}$ 'an, while the promotion of tourist attractions in Xi'an is mainly in Chinese, supplemented by English, and lacks Japanese propaganda, there are many problems in the Japanese introduction of scenic spots. This study investigates the Japanese signs of the nine scenic spots in Xi'an and the present situation of Japanese speakers. To collect and organize the Chinese presentations of the nine scenic spots, as well as other documents related to the scenic spots. This paper analyses the situation of Japanese translation of signs and placards of the nine scenic spots, as well as the present situation of Japanese interpreters' introduction to Japanese, including which are more standardized, which are problematic, and what are not. On the basis of the introduction of Japanese translation and Japanese introduction, this paper puts forward some suggestions on how to teach Japanese in future.
\end{abstract}

\section{Results of Research on Japanese Translation of Tourist Attractions in Xi'an}

As an important link in the development of the tourism culture industry in Xi'an, the tourist attraction is a key link in the development of the tourism culture industry in Xi'an and the development of Xi'an as a famous historical city, is a representative of the good image of the international metropolis of Xi'an. This subject introduces the Study of standardization through the Japanese translation of tourist attractions and the introduction of Japanese, it can reflect the historical significance, cultural quality and humanistic quality of the scenic spot correctly.

At present, due to the uneven level of translators, Chinese and Japanese language and culture and aesthetic differences, the quality of Japanese introduced in the scenic spots is not good. Based on this research, we have studied the Chinese character translation of Hua qing Pool, Giant Wild Goose Pagoda, Terracotta Army, Da Tang Fu Rong Yuan, Daming Palace, Drum Tower, Stele, Shaanxi Provincial Museum of History and Xi'an Ming City Wall, and an oral Japanese introduction by Japanese speakers.

Signs, Display Boards and Other Literal Japanese Translation

So far, the author has studied the present situation of the nine major scenic spots in Hugging Pool, Giant Wild Goose Pagoda, Terracotta Army, Da Tang Fu Rong Yuan, Daming Palace National Heritage Park, Clock Drum Tower, The Monument Museum, Shaanxi Provincial Museum of history, Xi'an Ming City Wall, and the present situation of Japanese introduction. It is found that there are not many Japanese signs and introduction of tourist attractions in Xi'an, and there are some problems such as non-standard and inconsiderate expressions in Japanese logo and Japanese introduction. At present, among the nine tourist attractions I have visited, the signs and the introduction are mainly in English, Japanese and Korean. The popularization rate of Japanese is $30 \%$, and that of Japanese is only $20 \%$. It can be seen that the popularization rate of Japanese in scenic spots and scenic spots is very low.

Among the nine scenic spots, the Xi'an Monument Museum is the most comprehensive in Japanese and Japanese translation. The guide plate and Japanese sign in the scenic spot are all equipped with Japanese translation, and the translation meaning is accurate. Detailed Japanese translations are included in the overall presentation of each of the booths. However, in the exhibition room, only the name of the Japanese translation of the name, did not translate the contents of the Japanese. However, the names of the exhibits and the presentation of the exhibits 
were translated into English. However, it is rare to have all the signage and guide signs translated in Japanese and the names of the exhibits are translated in Japanese.

The guide plates and signs of the National Heritage Site in Daming Palace are provided with Japanese translation. Some of the exhibits are presented in Japanese. Most of the exhibits are presented in English only. Xi'an Ming City Wall Guide Plate, signage only English, Korean translation, and no Japanese translation. The area and exhibits are only available for translation in English There is no Japanese translation. The guides, signs, areas and exhibits in the pagoda are translated only in English and do not have Japanese translation. The electronic self-service reading system in Giant Wild Goose Pagoda Tower is only available in Japanese and the content is detailed and translated accurately. However, the electronic self-service reading system is located only inside the Giant Wild Goose Pagoda. There is no Japanese translation of the outside of the tower and the signage, which makes it difficult for Japanese visitors, especially those who come to visit on their own. The guides, signs areas and exhibits of the Shaanxi Provincial Museum of History are translated only in English and do not have Japanese translation. The Terracotta Army, signage, area and exhibit descriptions are available in English only. The regional and exhibits descriptions are available in English only. There is no Japanese translation.

The use of Japanese signs can be divided into indicative, expository, restrictive and mandatory notices according to their indicative functions. At present, most of the Japanese signs in Xi'an scenic spot are about road signs and danger reminders, however, there are few Japanese signs in public service facilities, and there is a lack of Japanese signs about the relevant indicative public notice, such as the ticket office, the depository office and the name of the site. The introduction of Japanese as an indicative public notice is intended to give visitors an idea of what the site is about, its historical origins, its cultural significance etc. Therefore, the Japanese language should be used as much as possible. It shouldn't be a literal translation. However, at present, many of the translation of scenic spots is a mechanical and literal translation, which does not conform to the Japanese expression.

Secondly, Chinese is now using simplified Chinese characters, while Japanese still uses traditional Chinese characters, known as Japanese characters. Japanese tourists can not understand if the Japanese word for traditional Chinese characters is to be replaced with simplified Chinese characters arbitrarily. According to the author's visit, the Japanese character of the scenic spot will appear in the simplified Chinese characters, the main is that the site staff used the translation machine, and did not ask professional translators to translate the result. Simplified Chinese characters are still commonly used in word processing in translation machines. For example starboardを步いてください。“right side”、“step”For both Simplified Chinese characters, should be changed to“右側を歩いてください。”

At present, Japanese logo and Japanese introduction are inaccurate, inappropriate, and the frequency of errors in grammar have the following problems

The problem of literal Japanese translation in tourist attractions in Xi'an

First, there are few scenic spots with Japanese signs and Japanese introduction.

Second, the mechanical translation of word by word more,does not conform to the Japanese expression habit.

The Japanese speakers 'oral Japanese introduction in six of the nine scenic spots in the author's research are equipped with Japanese speakers. After recording the introduction of Japanese speakers, I find that there are two problems

\section{The self-made synthetic words.}

many words in Chinese do not use"of" can be expressed in the order of precedence, while in Japanese the word"の"must appear if it is not a compound word. If you don't use the word "の", it means that the word is an inherent synth in Japanese If the Japanese does not have such a compound word and arbitrarily removes the $の$, the translation of the word is wrong. For example, "Ming 
Dynasty Architecture" translates directly to "Ming Dynasty Architecture" , which should be translated as "Ming の Era の Architecture" .

\section{The mechanical transformation of Chinese characters}

The mechanical transformation of Chinese characters is to change the simplified Chinese characters directly into traditional Chinese characters, and then to use the pronunciation as Japanese. Refers to the conversion of simplified Chinese characters to traditional Chinese characters, which are then used as the Japanese language. For example, the "Confucius Temple" appeared in the Japanese introduction of the Museum of Inscriptions. It is clear that Confucius Temple is a mechanical transformation of the word Confucius temple into a traditional character "Confucius Temple", which is not explained, and for Japanese tourists who do not understand Chinese culture, it is difficult to understand the meaning of the word "Confucius Temple""Wu Zetian" translates as "Wu Zetian", while in Japanese"Wu Zetian" is usually translated as "empress tse-tung". As if it were a Japanese word, there is no such word in Japanese. It is the result of the mechanical transformation and straightforward translation of the Chinese"historical myth" .

\section{The Main Reasons for the Problems Arising from the Japanese Sign and the Japanese Introduction}

The author thinks there are three main reasons why the Japanese logo and the Japanese introduction have the problem.

First, the Japanese sign and the Japanese introduction were not paid enough attention in the individual scenic spots, and many sites did not have signs and scenic spots and Japanese translations of exhibits

Second, the Japanese speakers in the scenic spots need to improve their Japanese proficiency. Japanese interpreter is easy to be interfered with by the mother language, Japanese level needs to be improved. The above adverbs are caused by the low level of Japanese in the use of error frequency, self-made synthetic words, and Chinese characters.

\section{Solution}

First of all, the government organs should pay full attention to the Japanese logo and the Japanese introduction of the scenic spots. In order to promote the international construction of Xi'an, Xi'an government departments must pay more attention to the Japanese sign and the Japanese introduction in the scenic spots, and re-examine the importance of its international image to Xi'an. We will increase the popularization of multilingual translation of scenic spots and descriptions to meet the needs of international tourists.

In addition, the scenic spot itself should pay full attention to the Japanese sign and the Japanese introduction of the scenic spot. In the scenic spots will be the Japanese logo, the introduction of Japanese popularization, standardization. In particular, the tourist experience should be the center of the service fee collection, providing the service to their satisfaction, So the Japanese tourist's Japanese logo and the introduction of Japanese should also follow up. The existing problem tags should be corrected in a serious and timely manner, and those that have not yet been set should be set up as soon as possible.

To improve the Japanese language proficiency of Japanese speakers and to employ Japanese-speaking personnel who have been trained in Japanese in a professional way. Considering the difficulty of translation in the scenic area, the most direct way for the scenic spot to introduce Japanese is to seek help from the college Japanese teacher. A study group on Japanese-language presentations could also be set up to provide research funding and experts could be invited to serve as research team members.

\section{Guiding Opinions on the Japanese Teaching of Tour Guides.}

The following points should be noted in the course of Japanese teaching. 
The stylistic differences between Chinese and Japanese:When the Chinese introduce the scenic spots, they are influenced by ancient parallel prose in language form, they are neat and symmetrical, harmonious, rhetorical, four-character structure, read aloud, give people a beautiful enjoyment. Japanese prose is more rustic, bombastic, figurative, and rendered words much less than Chinese.

Cultural differences between China and Japan:Language is the response of the mind, and people are living in a certain social and cultural environment. Only if we fully understand and understand the social culture of Japan and grasp the essence of Japanese social culture, language will not remain on the surface of words. In the introduction of tourist attractions, especially the oral presentations in Japanese should take full account of the culture and customs of the target country and avoid the confusion caused by cultural differences as much as possible.

Differences in Chinese and Japanese aesthetics:Because of cultural differences, people of different languages have developed a kind of inherent aesthetic psychology and appreciation habit under the influence of their specific language and culture environment, this mentality and habits naturally lead them to form different writing styles and aesthetic standards. The language aesthetics of the Han nationality has always emphasized the unity of the "meaning and landscape"of landscape description, pursuing the high harmony of objective scenes and subjective emotions, and the beauty of unity. Japanese scenes are depicted without too much image rendering, and the language is more objective in expression, often conveying the real beauty with a straight line of figurines.

The study, based on a field trip to Xi'an' s main scenic spots, found problems in Japanese signs and Japanese-language presentations, and then analyzed the reasons for the problems. At last, several solutions and translation methods are put forward. It is hoped that through the research of this subject, it will promote the development of the tourism industry in Xi'an by further publicizing the culture of Xi'an and improving the understanding of Xi'an among Japanese tourists.

\section{Acknowledgement}

Project name: A standardized study on the introduction of Japanese in tourist attractions in Xi'an, Project No. : 14WL21

\section{References}

[1]. Yang Guomin. "Alienation""domestication"and the standardization strategy of tourist scene translation-from the public signs of Guangzhou Baiyun Mountain Scenic Area [J]. Journal of Social Sciences(Beijing Institute of Technology), 2007, (4) 109-1132.

[2]. Wang Qiusheng. The translation of tourist attractions needs to be standardized [J]. Translation of China, 2004. (03):77-793

[3]. Guo Dingqin. On the cultural implication in translation of tourist attractions[J]. Foreign Language and translation2007, 8(3):148-1494.

[4]. Liangchen. The translation of tourist attractions needs to be improved [J]. "LiteLi rature and teaching materials", 2007(01):137-1385.

[5]. Li Zaixia, Wang Shuling. Issues to be noted in Tourist Chengde Mountain Resort and its outlying temples translation-also on the Chengde Mountain Resort and its outlying temples scene. Chengde Journal of Advanced Petroleum Vocational School, 2008, 10(3):91-94

[6]. Dong Yajun Cui Yanrong Ge Yanrong. The promotion of tourism by cross-cultural Communication of tourism scenic spot translation[J]. "Modern shopping malls", 2008(20):243-244. 